JKL-14 Japan-Korea Joint Session of the 92nd Annual Meeting of the Japanese Pharmacological Society

\title{
The exposure of early life stress induce chronic pain mediating through decrease of opioidergic signaling in adulthood
}

\author{
Kazuo Nakamoto $^{1}$, Shogo Tokuyama ${ }^{1}$ \\ ${ }^{1}$ Department of Clinical Pharmacy, School of Pharmaceutical Sciences, Kobe Gakuin University, 1-1-3 Minatojima, \\ Chuo-ku, Kobe 650-8586, Japan
}

The experience of the severe stress in the childhood (early life stress) such as abuse or neglect is a significant risk factor for psychiatric disorders in adulthood. We have already developed early life stress model mice which subjected to maternal separation combined with social isolation (MSSI). These model mice showed emotional dysfunction and exacerbated nerve injury-induced mechanical allodynia leading to the chronic pain. However, these mechanisms remain unclear. In this study, we investigated the involvement of opioidergic signaling, a one of endogenous pain control system, in MSSI mice. In the periaqueductal gray (PAG) area, a region that is implicated in the opioid control of nociception, $\mu-, \delta$ - and $\kappa$-opioid receptor (MOR, DOR and KOR) mRNA expression were significantly decreased in MSSI mice compared to control mice. A large number of c-Fos, a neuronal activity marker, positive cells were observed in the PAG of MSSI mice injected with morphine, whereas control mice without morphine treatment are hardly detectable c-Fos positive cells. Furthermore, MSSI mice showed decrease of morphine analgesia in the tail flick test compared to control mice. Our findings suggest that early life stress may induce the development of chronic pain through the alteration of opioidergic neuron system in the PAG area. 